\title{
Formation of biomolecule precursors in space?
}

\author{
Wolf D. Geppert ${ }^{1}$, Erik Vigren ${ }^{1}$, Mathias Hamberg ${ }^{1}$, \\ Vitali Zhaunerchyk ${ }^{1}$, Magdalena Kaminska ${ }^{2}$, Richard D. Thomas ${ }^{1}$, \\ Fabian Österdahl ${ }^{1}$, Fredrik Hellberg ${ }^{1}$, Anneli Ehlerding ${ }^{1}$, \\ Mathias Danielsson ${ }^{1}$, and Mats Larsson ${ }^{1}$ \\ ${ }^{1}$ Physics Department, Stockholm University \\ Roslagstullsbacken 21, S-10691, Stockholm, Sweden \\ email: wgeppert@physto.se \\ ${ }^{2}$ Swietokrzyska Akademy, Swietokrzyska, PL-25406 Kielce,Poland
}

\begin{abstract}
The possibility of an extraterrestrial origin of biomolecule building blocks has been a subject of intense discussions for many years. The detection of amino acids in meteorites opens the possibility of a delivery of biomolecules synthesized in the interstellar medium or star-forming regions to the primeval Earth. Whereas it can be doubted if more complex species like amino acids can survive the strong UV radiation in the early Solar System, this does not necessary hold for more primitive precursor molecules like nitriles. These compounds can also be synthesized very efficiently in methane-nitrogen dominated atmospheres like the one present on Titan and the early ages of Earth. This Contribution focuses on the formation and degradation processes of nitriles in interstellar clouds and planetary atmospheres and on their possible role in the generation of biomolecules.
\end{abstract}

Keywords. Astrochemistry, molecular processes, plasmas, ISM: molecules, astrobiology

\section{Introduction}

One of the most interesting questions in astrobiology is to what extent the basic building blocks of life can be formed in the interstellar medium and from what level of complexity planetary conditions (dense atmospheres, oceans) are necessary. For a long time it was taken as certain that biomolecules were formed in a "primordial soup" under an atmosphere of water, ammonia and methane that was subject to high-voltage discharges, an environment mimicked by the famous Urey-Miller experiment (Miller 1953). There are two problems with that pathway of biomolecule formation: Firstly, amino acids are formed as racemates in the Urey-Miller reactor and, secondly, their synthesis is hindered by the presence of oxygen, whose abundance in the atmosphere of early Earth is still a subject of controversial discussion (Rosing \& Frei 2004). On the other hand, analysis of certain meteorites revealed the presence of several amino acids and investigations into the hydrogen isotope distribution suggested an interstellar origin of these compounds (Bernstein et al. 2002). Furthermore, some of the amino acids detected in the Murchison and Murray meteorite showed quite substantial enantiomeric excesses of the (biological) L-form (Pizarrello \& Cooper 2002, Cronin \& Pizarrello 1997), which raises the question if the first amino acids on Earth, and thus homochirality of life, could have an interstellar origin. The problem with that scenario is that amino acids cannot be expected to survive the strong UV field present in the early Solar System (Ehrenfreund \& Sephton 2006). This, however, does not hold for nitriles, which possess a much higher photostability than their corresponding acids (Bernstein et al. 2004) and can, after their arrival at planetary 
surfaces, hydrolyze to form amino acids or polymerize to tholines. Nitriles have been detected in several different interstellar objects, like circumstellar envelopes (Matthews \& Sears 1986) and dark clouds (Johansson et al. 1984). Interestingly, the largest molecule hitherto detected in the interstellar medium, $\mathrm{HC}_{11} \mathrm{~N}$, is a nitrile (Bell et al. 1982).

Nitriles are especially important in nitrogen-methane dominated atmospheres like those of Titan and the early Earth. In one of the flybys of the Cassini probe, much higher abundances of protonated nitriles than predicted by previous models have been discovered (Vuitton et al. 2006). It is very likely that nitriles are involved in the formation of the haze in Titan's ionosphere (Lavvas et al. 2008a), since copolymers of acetylene and $\mathrm{HC}_{3} \mathrm{~N}$ have been found to exhibit optical properties similar to that haze (Clarke \& Ferris 1997). In preparation for projected space research programs like the Titan Explorer mission it is of pivotal importance to elucidate the formation and destruction mechanisms of these species to understand the chemical processes in the ionosphere of Titan.

If one assumes that nitriles can be delivered to planets from the interstellar medium, the question of their formation pathway arises. In the gas phase, a synthesis involving ion-neutral reactions (e.g., radiative associations) leading to a protonated nitrile followed by a dissociative recombination (DR) that yields the unprotonated species and a hydrogen atom, is often invoked, as in the case of acetonitrile (Huntress \& Mitchell 1979).

$$
\begin{aligned}
& \mathrm{CH}_{3}^{+}+\mathrm{HCN} \rightarrow \mathrm{CH}_{3} \mathrm{CNH}^{+}+\mathrm{h} \nu(1) \\
& \mathrm{CH}_{3} \mathrm{CNH}^{+}+\mathrm{e}^{-} \rightarrow \mathrm{CH}_{3} \mathrm{CN}+\mathrm{H}(2)
\end{aligned}
$$

Another important mechanism for nitrile formation is the reaction of $\mathrm{CN}$ radicals with hydrocarbons (Herbst \& Leung 1990). In the case of acetylene and ethylene (Balucani et al. 2000a), such processes have experimentally been shown to lead to cyanoethylene $\left(\mathrm{HC}_{3} \mathrm{~N}\right)$ and acrylonitrile $\left(\mathrm{C}_{2} \mathrm{H}_{3} \mathrm{CN}\right)$, respectively.

$$
\begin{aligned}
& \mathrm{C}_{2} \mathrm{H}_{2}+\mathrm{CN} \rightarrow \mathrm{HC}_{3} \mathrm{~N}+\mathrm{H}(3) \\
& \mathrm{C}_{2} \mathrm{H}_{4}+\mathrm{CN} \rightarrow \mathrm{C}_{2} \mathrm{H}_{3} \mathrm{CN}+\mathrm{H}(4)
\end{aligned}
$$

In planetary atmospheres insertion reactions of nitrogen atoms in the long-lived excited $\left({ }^{2} \mathrm{D}\right)$ state are crucial to the formation of nitriles (Balucani et al. 2000b):

$$
\mathrm{C}_{2} \mathrm{H}_{4}+\mathrm{N}\left({ }^{2} \mathrm{D}\right) \rightarrow \mathrm{CH}_{3} \mathrm{CN}+\mathrm{H}(5)
$$

Due to their high proton affinity, nitriles can easily be protonated (in the interstellar medium by $\mathrm{H}_{3}^{+}, \mathrm{HCO}^{+}$and $\mathrm{N}_{2} \mathrm{H}^{+}$, in Titan's ionosphere by reactions with hydrocarbon ions), which explains the high nitrile abundances detected by the Ion Neutral Mass Spectrometer (INMS) on board the Cassini spacecraft. Since, according to models, abundances of many nitriles in Titan's atmosphere peak at an altitude of around $1000 \mathrm{~km}$ (Lavvas et al. 2008b), i.e., well into the ionosphere, protonation of nitriles is very probably an important decay process of nitriles there and could compete with polymerization and ionization by solar UV photons and magnetospheric electrons. DR is one of the most crucial destruction pathways of these protonated nitriles in interstellar clouds and planetary ionospheres, but, unfortunately, little experimental data are available on the rates and product branching ratios of these reactions. This Contribution sums up recent work 
performed by the Stockholm Group on the DR of protonated nitriles to address this lack of fundamental data.

\section{Experimental details}

The DR experiments have been performed at the heavy-ion storage ring CRYRING at the Manne Siegbahn Laboratory, Stockholm University. The experimental procedure has been described in detail elsewhere (Neau et al. 2000) and is therefore summarized only briefly here. For technical reasons related to the data analysis (better resolution on the detector) fully deuterated compounds were used in the case of protonated acetonitrile and protonated methylacetylene. After creation of the ions in the source they were further accelerated by a $\mathrm{RF}$ field to translational energies of $1.78 \mathrm{MeV}\left(\mathrm{C}_{2} \mathrm{H}_{3} \mathrm{CNH}^{+}\right.$and $\left.\mathrm{DCCCND}^{+}\right)$and $2.09 \mathrm{MeV}\left(\mathrm{CD}_{3} \mathrm{CND}^{+}\right)$, respectively. The ions were stored in the ring for several seconds to allow for cooling by radiative deactivation and superelastic collisions with electrons. The ion beam was then merged with a mono-energetic electron beam in an electron cooler, the length of the interaction region being $0.85 \mathrm{~m}$. Neutral products generated by DR reactions in the electron cooler left the ring tangentially and were detected by an energy-sensitive ion implanted silicon detector with a radius of $17 \mathrm{~mm}$ mounted at a distance of $3.85 \mathrm{~m}$ from the centre of the interaction region. A background signal due to neutral products emerging from collisions of the ions with residual gas was also present: this was measured with the relative kinetic energy between the ions and electrons tuned to 1 or $2 \mathrm{eV}$, where the DR cross section is very low and the measured neutral fragments are therefore almost exclusively produced by rest gas collisions. This background was subsequently subtracted from the total detector signal.

\section{Results and Discussion}

Cross sections and reaction rate constants - For the cross section measurements, the kinetic energy in the centre-of-mass frame between the ions and the electrons was changed between 1 and $0 \mathrm{eV}$ by ramping the cathode voltage of the electron cooler over a certain time interval (1-2 s) from a high value corresponding to a centre-of-mass energy of $1 \mathrm{eV}$, the electrons being faster than the ions, down to a low-value also corresponding to $1 \mathrm{eV}$ where the electrons were slower than the ions. Thus, a voltage corresponding to a centreof-mass energy of $0 \mathrm{eV}$ is reached during the scan. The signal from the detector was monitored by a single channel analyzer, selecting signals only when all the fragments reach the detector simultaneously, and thereafter recorded by a multichannel scaler, yielding the number of counts vs. storage time and, therefore, at a given relative kinetic energy. After correction for space charge, toroidal effects, and the energy spread of the electrons (see, e. g., Geppert et al. 2006 for details), the cross sections of the investigated DR reactions could be determined. Under assumption of a Maxwell-Boltzmann distribution of kinetic energies of the reactants, their thermal rate constants were calculated. Both cross sections and rate constants are listed in Table 1.

Branching fractions - The fragments produced by a DR event reached the detector within a very short time interval compared with its response time. The pulse height of the detector signal was therefore proportional to the total mass. To measure the branching fractions a metal grid with a transmission $T=0.297 \pm 0.015$ was inserted in front of the detector (Neau et al. 2000). In this case the registered DR spectrum splits into a series of peaks with different energies corresponding to the sum of the masses of the particles passing the grid. Unfortunately, for the investigated ions, the resolution of the detector did not allow a separation of fragment masses differing only by a the mass of a $\mathrm{D}$ or $\mathrm{H}$ atom, so only conclusions about the probabilities of ruptures of bonds between the heavy atoms could be obtained. The branching fraction for the different break-up 
Table 1. Reactive cross sections and thermal rate constants of the DR of investigated protonated nitriles.

\begin{tabular}{|c|c|c|c|}
\hline Ion & Cross section $/ \mathrm{cm}^{2}(\mathrm{E}$ in $\mathrm{eV})$ & Rate constant $/ \mathrm{cm}^{3} \mathrm{~s}^{-1}(\mathrm{~T}$ in $\mathrm{K})$ & Reference \\
\hline $\mathrm{D}^{+}$ & $2.3 \pm 0.4 \times 10^{-15} \mathrm{E}^{-1.10 \pm 0.02}$ & $1.5 \pm 0.3 \times 10^{-6}(\mathrm{~T} / 300)^{-0.58 \pm 0.02}$ & $\begin{array}{c}\text { Geppert et al. } \\
(2004)\end{array}$ \\
\hline $\mathrm{CD}_{3} \mathrm{C}$ & $7.4 \pm 1.5 \times 10^{-16} \mathrm{E}^{-1.23} \pm 0.02$ & $8.1 \pm 1.6 \times 10^{-7}(\mathrm{~T} / 300)^{-0.69} \pm 0.02$ & $\begin{array}{c}\text { Vigren et al. } \\
(2008)\end{array}$ \\
\hline $\mathrm{C}_{2} \mathrm{H}_{3} \mathrm{CNH}^{+}$ & $1.1 \pm 0.2 \times 10^{-15} \mathrm{E}^{-1.29} \pm 0.02$ & $1.8 \pm 0.4 \times 10^{-6}(\mathrm{~T} / 300)^{-0.80 \pm 0.02}$ & $\begin{array}{l}\text { Preliminary } \\
\text { data }\end{array}$ \\
\hline
\end{tabular}

channels have been computed using a simple Gaussian elimination process (see Geppert et al. 2004 for details). In the case of $\mathrm{DCCCND}^{+}$, the probability of the retention of the carbon chain is $52 \pm 5 \%$, and the rest of DR processes leads to fragmentation of the central C-C bond. For $\mathrm{C}_{2} \mathrm{H}_{3} \mathrm{CNH}^{+}$the branchings fractions of these two pathways are equal (50\%). Interestingly, no rupture of the $\mathrm{C}-\mathrm{N}$ or the terminal $\mathrm{C}-\mathrm{C}$ bond is observed for both ions. In $\mathrm{CD}_{3} \mathrm{CND}^{+} \mathrm{DR}$ leads to conservation of the $\mathrm{C}-\mathrm{CN}$ chain in $65 \%$ of all events. The remainder result in the break-up of one C-N or C-C bond. Fragmentation of more than one heavy atom bond was not observed in any cases.

These results would imply that the sequence of Reactions (1) and (2) is a feasible pathway for synthesis of acetonitrile in dark interstellar clouds. However, it has been revealed in a FT-ICR experiment that the product of the first process is not protonated acetonitrile but a highly excited state of protonated methyl isonitrile $\left(\mathrm{CH}_{3} \mathrm{NCH}^{+}\right)$, which can either radiatively decay or convert to $\mathrm{CH}_{3} \mathrm{CNH}^{+}$through collisions (Anicich et al. 1995). Nevertheless, the latter process is too slow to be of any significance in the interstellar medium or planetary ionospheres. A way out could be the reaction of methyl cations with HNC, of which considerable densities are found in dark clouds, especially in TMC-1 where it is more abundant than HCN (Pratap et al. 1997):

$\mathrm{CH}_{3}^{+}+\mathrm{HNC} \rightarrow \mathrm{CH}_{3} \mathrm{CNH}^{+}+\mathrm{h} \nu(6)$

The degradation of nitriles through the protonation - DR sequence partly leads to recovery of the nitriles, but also results in formation of reactive products, which can undergo further chemical reactions. Since the reaction rates obtained for the investigated nitriles are in the upper range of those usually found in DR reactions [in the case of protonated acetonitrile they are about a factor of 2.5 higher than previous flowing afterglow measurements (Geoghegan et al. 1991)], they could have a far-reaching influence on the chemistry of nitrogen compounds in the interstellar medium and planetary ionospheres. Future model calculations incorporating these new data will show what impact our results have on the predictions of molecular abundances emerging from such computations.

\section{Acknowledgements}

The authors want to thank the staff at Manne Siegbahn Laboratory for excellent technical support. Funding from the Swedish Space Board and the Swedish Research Council is gratefully acknowledged.

\section{References}

Anicich, V. G., Sen, A. D., Huntress, W. T., \& McEwan, M. 1995, J. Chem. Phys., 102, 3256 Balucani, N., Asvany, O., Huang, L. C. L., Lee, Y. T., Kaiser, R. I., Osamura, Y., \& Bettinger, H. F. 2000a, ApJ, 545, 892 
Balucani, N., Cartechini, L., Alagia, M., Casavecchia, P., \& Volpi, G. G. 2000b, J. Phys. Chem. A., 104, 5655

Bell, M. B., Feldman, P. A., Kwok, S., \& Matthews, H. E. 1982, Nature, 295, 389

Bernstein, M. P., Dworkin, J. P., Sandford, S. A., Cooper, G. W., \& Allamandola, L. J. 2002, Nature, 416, 401

Bernstein, M. P., Ashbourn, S. F. M. Sandford, S. A., \& Allamandola, L. J. 2004, ApJ, 601, 365

Clarke, D. W. \& Ferris, J. P. 1997, Icarus, 127, 158.

Cronin, J. R. \& Pizarrello, S. 1997, Science, 295, 951

Ehrenfreund, P. \& Sephton, M. A. 2006, Faraday Disc., 133, 277

Geoghegan, M., Adams, N. G., \& Smith, D. 1991, J. Phys. B., 24, 2589

Geppert, W. D., Ehlerding, A., Hellberg, F., Semaniak, J., Österdahl, F., Kaminska, M., AlKhalili, A., Zhaunerchyk, V., Thomas, R., af Ugglas, M., Källberg, A., Simonsson, A., \& Larsson, M. 2004, ApJ, 613, 1302

Geppert, W. D., Hamberg, M., Thomas, R. D., Österdahl, F., Hellberg, F., Zhaunerchyk, V., Ehlerding, A., Millar, T. J., Roberts, H. Semaniak, J., af Ugglas, M., Djuric, N., Källberg, A., Simonsson, A., Kaminska, M., \& Larsson, M. 2006, Faraday Disc., 133, 177

Herbst, E. \& Leung, C. M. 1990, A\&A, 233, 177

Huntress, W. T. \& Mitchell, G. F. 1979, ApJ, 231, 456

Johansson, L. E. B., Andersson, C., Elldér, J., Friberg, P., Hjalmarsson, A., Höglund, B., Irvine, W. M., Olofsson, H., \& Rydbeck, G. 1984, A\&A A, 130, 227

Lavvas, P. P., Coustenis, A., \& Vardavas, I. M., 2008a, Planet. Space Sci., 56, 27

Lavvas, P. P., Coustenis, A., \& Vardavas, I. M., 2008b, Planet. Space Sci., 56, 67

Matthews, P. \& Sears, T. J. 1986, ApJ, 300, 766

Miller, S. L. 1953, Science, 117, 528

Neau, A., Al-Khalili, A., Rosén, S., Le Padellec, A., Derkatch, A. M, Shi, W., Vikor, L., Larsson, M., Nagard, M. B., Andersson, K., Danared, H., \& af Ugglas, M. 2000, J. Chem. Phys., 113,1762

Pizarrello, S. \& Cooper, G. W. 2001, Meteoritics and Planetary Science, 36, 897

Pratap, P., Dickens, J. E., Snell, R. L., Miralles, M. P. Bergin, E. A., Irwine, W. M., \& Schloerb, F. P. 1997, ApJ, 486, 862

Rosing, M. T. \& Frei, R. 2004, Earth Planet. Sci. Lett., 217, 234

Vigren, E., Kaminska, M., Hamberg, M., Zhaunerchyk, V. Thomas, R. D., Danielsson, M. Semaniak, J. Andersson, P. U., Larsson, M, \& Geppert, W. D., 2008 Phys. Chem. Chem. Phys., 10, 4014

Vuitton, V., Yelle, R. V., \& Anicich, V. G. 2006, ApJ (Letters), 647, L175

\section{Discussion}

BERNSTEIN: It's interesting that when you have the protonated acrylonitrile you get the acrylonitrile product half of the time and half of the time you get carbon bond fragmentation. When the protonated acrylonitrile fragments, what other products do you get? Does that give you acetonitrile ultimately? That's a very interesting pathway as well.

GEPPERT: I think that is an important point. You unfortunately cannot work out the complete reaction scheme, so you cannot really make any conclusions about what products you get. The upper limit of recovery of acrylonitrile is, as said, about $50 \%$. Unfortunately there are many different exoergic pathways for breaking up after recombination. Amongst the events which involve a break up of a bond between two heavy atoms I would assume that a lot goes into $\mathrm{HNC}$, and $\mathrm{C}_{2} \mathrm{H}_{3}$ (which is a comparatively stable radical) but I would not bet my (comparatively mundane) salary on it. 


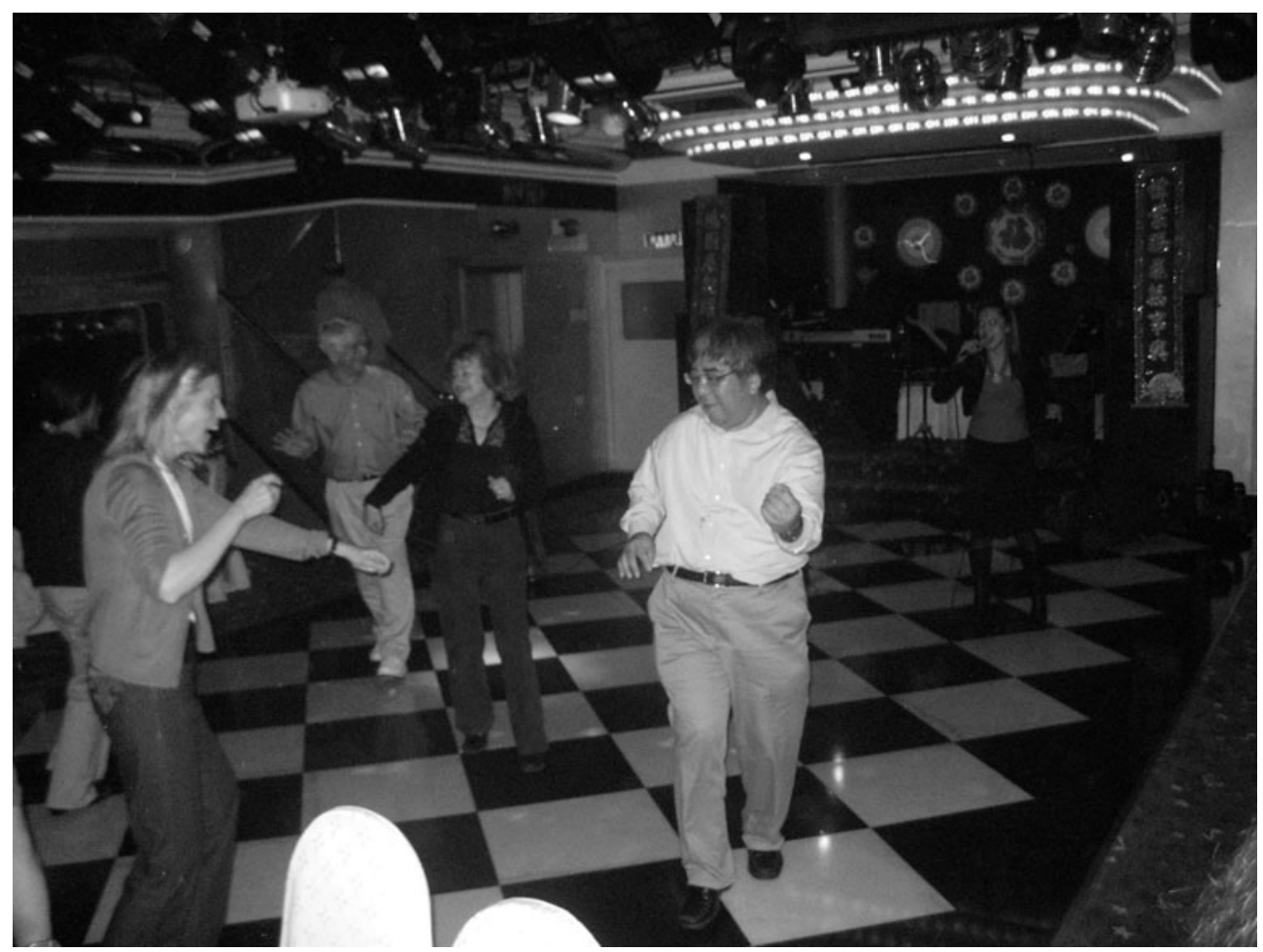

Dancing on board the Bauhinia during the harbour cruise (from left to right: Lucy Ziurys, Peter Strittmatter, Catherine Cesarsky, and Sun Kwok) (photo by Bruce Hrivnak). 